\title{
Violencia en la familia y en la relación de pareja en universitarios de Osorno, Chile
}

\author{
Soraya Espinoza Moraga \\ Universidad de Los Lagos, Osorno, Chile. \\ Email: sespinoza@ulagos.cl \\ Ramón Vivanco Muñoz \\ Universidad de Los Lagos, Osorno, Chile. \\ Email: rvivanco@ulagos.cl \\ Alex Veliz Burgos \\ Universidad de Los Lagos, Osorno, Chile. \\ Email: alex.veliz@ulagos.cl

\section{Antonio Vargas Peña} \\ Universidad de Los Lagos, Osorno, Chile. \\ Email: antoniovargaspena@gmail.com
}

\begin{abstract}
Resumen: La violencia de género no deja indiferente a ninguno de sus miembros, especialmente, a los hijos/as, además aumenta el riesgo del maltrato contra ellos. Dentro de sus implicancias esta la violencia en etapas posteriores. Esta investigación busca describir la relación entre dichas experiencias y ejercer o ser víctima de violencia en sus relaciones románticas en universitarios. Se les aplicó un cuestionario de Violencia en pareja yEscala de Maltrato a una muestra de 360jóvenes. Los resultados indican una relación significativa en hombres y mujeres que declaran promedios más altos en violencia psicológica, emocional y física. La experiencia de violencia de género psicológica tendría mayor repercusión en hombres, mientras que el maltrato desde los padres tendría mayor repercusión en las mujeres.
\end{abstract}

Palabras clave: Violencia de género; maltrato infantil; violencia en noviazgo

\section{Violence within the family and in the intimate partner relationship among university students in Osorno, Chile}

\footnotetext{
Abstract: Gender violence does not leave any of its members indifferent, especially children, and it also increases the risk of mistreatment against them. Among its implications is violence in later stages. This research seeks to describe the relationship between these experiences and exercise or be a victim of violence in their romantic relationships in college. A questionnaire of Violence in Couple and Scale of child Abuse was applied to a sample of 360 young people. The results indicate a significant relationship in men and women who declare higher averages in psychological, emotional and physical violence. The experience of psychological gender violence would have greater impact on men, while abuse from parents would have greater impact on women
}

Keywords:Gender violence; child abuse; dating violence 


\section{Violência na família e na relação de casais entre universitários de Osorno, Chile.}

Resumo: A violência de gênero não deixa nenhum de seus membros indiferentes, especialmente crianças, e também aumenta o risco de maus-tratos contra eles. Entre suas implicações está a violência em fases posteriores. Esta pesquisa busca descrever a relação entre essas experiências e exercícios ou ser vítima de violência em seus relacionamentos amorosos na faculdade. Um questionário de Violência em Casal e Escala de Abuso foi aplicado a uma amostra de 360 jovens. Os resultados indicam uma relação significativa entre homens e mulheres que declaram médias mais altas em violência psicológica, emocional e física. A experiência da violência psicológica de gênero teria maior impacto sobre os homens, enquanto o abuso dos pais teria maior impacto sobre as mulheres.

Palavras chave: Violência de gênero; abuso infantile; violência no namoro

\section{Introducción}

Es en 1979 que desde la Asamblea General de la ONU se declara la eliminación de todas las formas de discriminación contra la mujer. Dicha convención representa la Carta Magna de los Derechos Humanos de la Mujer. Desde entonces y paulatinamente se toma conciencia y se otorga un tratamiento específico para la violencia contra las mujeres. Es así que en 1993 la ONU establece la declaración sobre la eliminación de la violencia de género, definiendo a esta última como

"Todo acto de violencia basado en la pertenencia al sexo femenino que tenga o pueda tener como resultado un daño o sufrimiento físico, sexual o psicológico para la mujer; inclusive las amenazas de tales actos, la coacción o la privación arbitraria de la libertad, tanto si se producen en la vida pública o privada" (ONU, articulo 1, 1993).

La violencia de género está vinculada a la desigual distribución del poder y las relaciones asimétricas que establecen entre mujeres y hombres, las cuales desvalorizan lo femenino y aseguran la subordinación hacia lo masculino (Brañes, 2006). La dominación masculina, como diría Bourdieu:

“[es] el producto de un trabajo continuado (histórico por lo tanto) de reproducción al que contribuyen unos agentes singulares (entre los que están los hombres consus armas como la violencia física y simbólica) y unas instituciones: familia, Iglesia, Escuela, Estado.(Bourdieu, 1998, p. 50)

El efecto de esta dominación no se produce de manera consciente o lógica, sino a través de esquemas de percepción, de apreciación que constituyen los hábitos y tiene efectos duraderos del orden social (Ibid). La familia agrupa todas las formas de violencia que ejerce el hombre sobre la mujer por su rol de género (Andrés, López y Álvarez, 2008). Además, entre el 30 y 60 \% de las familias en las que el hombre maltrata a su cónyuge o pareja, las hijas o hijos son también víctimas directas del maltrato (Edleson, 1999). La violencia de género es una realidad reconocida tanto en el ámbito internacional como nacional, reconociéndose tasas muy altas de prevalencia, sin embargo mucha menos atención se presta a los niños y niñas expuestos a esta violencia, más a aún si a eso le sumamos que un número significativo de niños/as experimentan situaciones de maltrato directo de sus padres.

Este sistema relacional se incorpora a partir de ritos, creencias, símbolos, valores. El hombre como ser social y la persona como actor social es un producto de los procesos de socialización de género que tiene lugar a lo largo de toda la vida y que conlleva la interiorización y/o práctica de roles diferenciados en función del sexo asignado al nacer (Matud, 2012). Uno de sus efectos importantes es concebir como algo natural el ejercicio de la violencia en las relaciones de noviazgo. Como parte de sus vivencias familiares, los jóvenes estarían más acostumbrados a este tipo de interacción (Gálligo, 2009)

Las investigaciones llevadas a cabo por la ONU (2006) y por el Consejo de Europa (2010)concluyen que los niños y niñas sufren de manera directa las consecuencias, no sólo físicas y emocionales de las situaciones de violencia en su hogar, sino también las derivadas de haber vivido y formado su personalidad en un 
ámbito de desigualdad de poder y sometimiento de la madre a la conducta violenta de un hombre, lo que potencialmente les convierte en elementos de la cadena de reproducción de esta violencia.

\section{Efectos de l exposición a la violencia de género}

El reconocimiento de los efectos perjudiciales sobre los niños/as expuestos a la violencia de género ha conllevado la necesidad de reconocerlos como sujetos activos de protección frente a esta realidad. Como principal herramienta normativa a nivel internacional se plantea la Convención de Naciones Unidas de los derechos del Niños y Niñas (CDN).Que Chile ratifica en el año 1999. Estudios que han realizado un meta - análisis de la prevalenciade violencia de género concluyen que las consecuencia negativas de la violencia observada son similares a las provocadas por el maltrato infantil (Kitzmann et al, 2003) y que la unión de ambas circunstancias constituye un factor aún más potente de riesgo ante estas consecuencias negativas (Hotaling y Sugarman, 1986; MacEwen, 1994).

Según los datos aportados por la UNICEF (United Nations International Children’s Emergency Fund) (2006) se estima que al menos 275 millones de niños y niñas en el mundo han sido testigos de violencia en su familia cada año, y además se afirma que "la violencia dentro de la pareja hace aumentar el riesgo de violencia contra los niños en el seno familiar” (P.16).En Estados Unidos los estudios de McCloskey y Walker (2000) señalan que entre un 20 y un $25 \%$ de los niños y niñas en edad escolar han sido testigos de violencia física entre sus padres. En España se estima, según la UNICEF y BoduShop en su estudio "Tras las puertas cerradas" (2006),que 188.000 niños y niñas estuvieron expuestos a violencia de genero. El 85\% fueron testigos de los malos tratos hacia sus madres y el $66 \%$ de los casos también ellos fueron maltratados.

\section{Maltrato infantil}

Sobre el maltrato en Chile, destaca el estudio realizado por Larrain y Bascuñan (2008) del tercer estudio de maltrato infantil de la UNICEF, concluye que el año 2006 en Chile, el75\% de los niños y niñas ha sufrido algún tipo de maltrato por parte de sus padres, un 51,5\% ha recibido violencia física, de estas un $25,6 \%$ fue violencia física leve y un 25,9\% violencia física grave.

Grupos de estudios longitudinales han reportado que los niños y adolescentes expuestos a violencia en la familia tiene más riesgo de desarrollar problemas en la niñez, adolescencia y adultez, los que incluyen agresividad, delincuencia, crimen, depresión, ansiedad, abuso de sustancias, problemas en las relaciones románticas y con los pares (McCloskey, 2011). Otro estudios señalan problemas interiorizados y exteriorizados, dificultades para relaciones sociales y utilización de estrategias agresiva en la resolución de problemas (Magen, 1999) y a justificar el uso de la violencia en sus relaciones amorosas (Lichter y McCloskey, 2004).

\section{Exposición a la violencia y violencia en el noviazgo.}

Algunos autores consideran a la violencia en las relaciones de noviazgo de adolescentes y jóvenes como un puente de unión entre la observación de la violencia en las familia y la violencia adulta o domestica (Makepeace, 1981; Bernard y Bernard, 1983; Guite, 2001). Un importante grupo de estudios han llegado a la conclusión que un porcentaje importante de personas que ejerció o recibió violencia en su relación de pareja, presenció dicha violencia entre sus padres durante su infancia o adolescencia. En adultos destacan los estudios (FernándezMontalvo y Echeburúa, 1997; Klevens, 2001; Langhinrichsen-Rohling, Neidig, y Thorn.1995; Matud et al., 2003; Rey, 2002; Rubiano, et al, 2003), y en adolescentes los estudios (Vg., Foshee, Bauman y Linder, 1999; Wolfe, et al, 2001). Un grupo importante de investigaciones confirman que la observación de violencia de los progenitores aumenta el riesgo de ejercer o sufrir violencia en las relaciones de pareja, en la etapa de la adolescencia (Kaukinen, 2014; Temched et al., 2008; Vagi et al., 2013)

Un estudio que evaluó a 8.629 niños/as expuestos a violencia de género y en particular violencia física hacia la madre, encontró que los menores expuestos eran más propensos a emplear la violencia en la pareja intima (Whitfield, et al, 2003). 
Por otra parte, el estudio de Ribero y Sánchez (2005) concluye que las mujeres provenientes de hogares en las cuales la madre era golpeada por el padre tienen mayores probabilidades de sufrir agresiones por parte de su pareja, debido a que asimilan una visión en la cual los maltratos son tolerables en el ámbito de la familia y en la que el padre es una figura dominante con capacidad de usar la violencia para imponer autoridad. Un estudio de Rey Anacona (2008), en una muestra de 105 adolescentes, encontró que los y las adolescentes que presenciaron violencia del padre hacia la madre se adjudicaban más rasgos de personalidad considerados machistas y sumisos, que los que no presenciaron violencia de género. Además mostraron estar más de acuerdo con una de las catorce afirmaciones que se utilizaron para evaluar la aceptación del uso de la violencia hacia la mujer. En este sentido, Meltzer, et, al (2009), concluyen que haber presenciado violencia doméstica puede enseñar a los niños/as que la violencia es una forma aceptable de resolver los conflictos entre los padres y predice la violencia de pareja en la adultez temprana así como que la violencia se interioriza como una estrategia de manejo del estrés adecuada (Loise, 2009).

Otro estudio de González y Fernández (2012) encontró que más del 66\% de hombres y mujeres justificaban los actos violentos, considerándolos necesarios y normales, ya que lo reconocen como algo aprendido desde la niñez.

En Colombia, Martínez, Vargas y Novoa(2016) encontraron que el 43.5\% (256 jóvenes) reportaron haber observado violencia entre sus padres y haber sido víctimas de algún tipo de violencia por parte de su pareja y el 8.3 \% (49 jóvenes) reportaron haber observado violencia entre sus padres y no informaron conductas de violencia de ningún tipo en su relación por parte de su novio o novia. También se halló una relación significativa entre haber observado violencia entre sus padres e informar el uso de al menos una conducta de maltrato por parte de la pareja. De igual forma, encontraron una relación significativa entre haber presenciado violencia entre los padres y haber cometido conductas de violencia en su relación de noviazgo. Entre las variables de la socialización temprana, la experiencia de abandono de la niñez y el haber presenciado violencia doméstica se relacionaron significativamente con la victimización y la perpetración. Cuando los participantes enfrentaron niveles más altos de agresión psicológica, los factores adversos de la socialización temprana se asociaron con niveles más altos de victimización y perpetración de violencia de pareja (Paat y Markham 2016). Desde el modelo de resiliencia se reconoce también que un grupo de ellos logra superar esta adversidad y evolucionan como adolescentes y adultos sanos (Martin, Best y Garmzy, 1991; Werner y Smith, 1989). No obstante, la ausencia de problemas no necesariamente significa que no hayan sido afectados, también influye los distintos niveles de exposición a esa violencia (Graham-Bermann et al., 2009) o la presencia de factores protectores que influyen sobre la magnitud de los efectos (DuMont, Widom y Czja, 2007; Luthar, Cicchetti y Becker 2000; Mc Gloin y Widom, 2001), como también del género del observador y del modelo, del tipo de rol jugado en la violencia marital adulta y del tipo de implicación en la violencia de los padres (Observación y/o ser objeto de maltrato) (Barnett, Miller-Perrin y Perrin 1997).Si bien existe una conexión entre la exposición a la violencia en la familia y la violencia en las relaciones de pareja, la transmisión inter generacional de la violencia no es universal (Cascardi, y Jouriles, 2018)

\section{Violencia en relaciones de parejas}

La violencia en el noviazgo (asimilable a pololeo en Chile, expresión proveniente del Mapudungun "Pulomen", Rodriguez, 1875) ha sido definida como actos de control, caracterizados por comportamientos agresivos que ocurren en una relación romántica entre parejas que no conviven, con el objeto de controlar o dominar a la persona física, sexual o psicológicamente (Close, 2005; Rey-Anacona, 2008). La violencia en la relaciones de parejas jóvenes es uno de los problemas sociales relevantes en la actualidad por el impacto en la salud tanto física como mental de los involucrados, como por el riesgo de que se transforme en un modelo estable de conducta que después se traspase a la vida conyugal y luego familiar(O’Leary, et, al,1989; Pederson y Thomas 1992). Las dimensiones del maltrato en el noviazgo se categorizan en maltrato físico, psicológico, sexual y económico (Rey, 2008; Osorio, 2013), y también contemplan las influencias socioculturales y los patrones familiares de violencia como parte del fenómeno (Osorio, 2013). En los últimos años se ha incrementado el interés por conocer los factores de riesgo de los actos de maltrato y violencia que ocurren en las relaciones de pareja de adolescentes y jóvenes que no se encuentran casados ni en relación de convivencia (Centers for Disease Control and Prevention, 2006; Corral, 2009; Lewis y Fremouw, 2001, Matud, 2007). 
La violencia en las relaciones de parejas adolescentes y jóvenes en los últimos estudios señalan una prevalencia preocupante. De 113 estudios revisados por Rubio Garay (2017)a nivel mundial los rangos en modalidades de violencia cometida y violencia sufrida se concluye que la violencia física cometida fluctúa entre un $7,7 \%$ y un $40,3 \%$ en los hombres frente a un 3,8\% y $41,9 \%$ en mujeres. En cuanto a sufrir violencia física, los rangos van de $0,4 \%$ a $53,7 \%$ en los hombres y de $1,2 \%$ a $41,2 \%$ para las mujeres. Para la violencia psicológica cometida los rangos de prevalencia figuran entre los 4,3\% a 95,3\% para los hombres y entre un $4,2 \%$ y un $97 \%$ en mujeres. Para violencia psicológica sufrida, los datos arrojan una variabilidad de $8,5 \%$ a $94,5 \%$ en hombres y de un $9,3 \%$ a un $95,5 \%$ en mujeres. En violencia sexual cometida la prevalencia fluctúa entre $2,6 \%$ a $58,8 \%$ en los hombres frente a un 1,2 a $40,1 \%$ en las mujeres y por último en esta misma violencia pero sufrida los rangos son de $0,1 \%$ a $54,2 \%$ para los hombres y de $1,2 \%$ a $64,6 \%$ para la mujeres.

Los datos de prevalencia también indican que en el noviazgo los niveles de violencia son semejantes entre ambos sexos. Sin embargo esta característica cambia en la relación de convivencia o matrimonio, pasando a ser la mujer la que es mayoritariamente objeto de violencia por parte de su pareja. Una hipótesis posible es que durante matrimonio o convivencia por diversos factores que limitan un desarrollo en condiciones de igualdad (menor salario, responsabilidad en el cuidado los/as hijos/as, menor integración laboral, menor participación en puestos de responsabilidad política y social), se da paso al establecimiento de relaciones de pareja inequitativas y desiguales en la distribución del poder no así en el noviazgo donde tendrá la mujer mayores grado de libertad (SERNAM,2009)

La familia es una institución más de la reproducción social,de la subordinación y violencia de género del cual nuestros jóvenes no son conscientes de su incorporación. La alta tasa nos plantea una situación no como casos aislados, sino como problema social, y en ese ámbito específico de la violencia de pareja se ha establecido que los implicados muestran de forma frecuente que han estado expuestos a violencia de género en la niñez y maltrato de sus padres.

Por lo anterior, esta investigación se planteó como objetivo describir como la violencia de parejas jóvenes en sus diferentes tipos interactúa con haber estado expuesto a violencia de género y maltrato en la niñez. Se trabajó con un grupo de adolescentes y jóvenes varones y mujeres que habían presenciado estos tipos de violencia en sus familias, comparándolos, tanto a nivel grupal como por género, con un grupo de adolescentes varones y mujeres con rasgos sociodemográficos similares, que no habían sido expuestos a ese tipo de violencia o de maltrato hacía ellos. En cuanto a las hipótesis se esperaba que los participantes expuestos a violencia de género o maltrato infantil presentaran mayor frecuencia de violencia en sus relaciones de pareja en los hombres y mayor victimización en mujeres que aquellos no tuvieron esa exposición.

\section{Métodos}

La muestra estuvo constituida por 360 casos (136 hombres y 224 mujeres) de estudiantes de cuatro Centros de Educación Superior de la ciudad de Osorno. Se seleccionaron estudiantes de ambos géneros ( $M=23,13$ años y DT= 4,4 años)a través de un muestreo incidental con participación voluntaria, firma de consentimiento informado y asegurando el anonimato de sus respuestas. Se utilizaron 2 instrumentos de recolección de información, el primero denominado Cuestionario de Violencia y Salud en Relaciones de Parejas Jóvenes. Este se basó en el protocolo básico del estudio multinacional de la OMS "Salud de la Mujer y Violencia Intrafamiliar" y que fue adaptado en Chile por el Centro de Políticas Públicas de la Universidad de Chile. Se extrajeron de este instrumento seis preguntas dicotómicas específicas referidas a la experiencia de violencia de género, (la ejercida del padre hacia la madre)y maltrato en la infancia entendida esta como la perpetrada por algún miembro de la familia. El segundo instrumento correspondió a una versión adaptada de la Lista de Chequeo de Experiencias de Maltrato en la Pareja -Forma A, referidas a violencia en relaciones de pareja de tipo psicológica, emocional, física, económica y sexual (Rey-Anacona, 2009). De este último instrumento se analizaron las subescala violencia psicológica, emocional y física compuesta por un total de 63 ítems. Las respuestas fueron medidas a través de una escala tipo Likert con las opciones "Nunca”, "Una vez", "Algunas veces” y "Muchas veces”. La adaptación del cuestionario consistió en su duplicación para cuantificar tanto perpetración como victimización de violencia en la relación de pareja (el cuestionario original sólo considera victimización), don- 
de cada ítem tuvo una doble entrada referida a la percepción de maltrato tanto ejercido como recibido respecto a o de su compañero/a.

Para la recolección de datos se tomó contacto con los estudiantes en los centros educacionales previa autorización de las autoridades de cada casa de estudios sumado a un consentimiento informado de parte de los jóvenes. Se aplicó un filtro en la selección de los/as estudiantes consistente en el reconocimiento por parte de estos/as de algún tipo de relación de pareja en los últimos 12 meses (pololeo).

\section{Análisis de datos}

Un primer grupo de técnicas estadísticas fueron los análisis de fiabilidad de las escalas y subescalas utilizadas a través del Alfa de Cronbach. En cuanto a las diferencias de medias entre hombres y mujeres, se contrastó por medio de la prueba t de Student para muestras independientes. Los cálculos se realizaron con SPSS16.

\section{Resultados}

Para validar los resultados se verificó la confiabilidad de las subescalas según la prueba estadística Alfa de Cronbach, dando como resultado valores que oscilan entre0.71 a 0.93(Tabla 1),lo que según Nunnally (1987) es adecuado pues para efectos de investigación pueden utilizarse escalas con valores mínimos de fiabilidad de 0.50 en adelante.

Tabla 1. Confiabilidad de escala de maltrato en la pareja forma A (Rey-Anacona, 2009)

\begin{tabular}{|l|c|}
\hline Subescalas & Alfa de Cronbach \\
\hline Violencia recibida (escala total) & 0.94 \\
\hline Violencia psicológica recibida & 0.93 \\
\hline Violencia emocional recibida & 0.77 \\
\hline Violencia física recibida & 0.72 \\
\hline Violencia ejercida (escala total) & 0.93 \\
\hline Violencia psicológica ejercida & 0.92 \\
\hline Violencia emocional ejercida & 0.72 \\
\hline Violencia física ejercida & 0.71 \\
\hline
\end{tabular}

Fuente: Vivanco, Espinoza, Romo, Veliz y Vargas (2015).

Como se observa en la tabla 2, 2 de cada 4 estudiantes mujeres del estudio señaló la presencia de violencia de género, destacándose que un 39,4\% reconoce que existió violencia psicológica entre sus padres, seguida de un $37,6 \%$ que manifiesta que escucho o vio violencia psicológica del padre a la madre.

En el caso de los hombres, ocurre algo similar, los porcentajes altos corresponden a la existencia de violencia psicológica $(33,9 \%)$ y ver o escuchar violencia psicológica(31,8\%). No obstante las mujeres reportaron percibir, ver o escuchar con mayor frecuencia respecto a sus pares hombres.

Tabla 2. Violencia de género reportada por estudiantes universitarios

\begin{tabular}{|l|c|c|}
\hline Violencia de género & Mujeres\% & Hombres\% \\
\hline Existió violencia física & 26.6 & 18.3 \\
\hline Vio o escuchó violencia física & 25.4 & 20.4 \\
\hline Existió violencia psicológica & 39.4 & 33.9 \\
\hline Vio o escucho violencia psicológica & 37.6 & 31.8 \\
\hline
\end{tabular}

Fuente: Elaboración Propia 
Tabla 3. Maltrato recibido reportado por estudiantes universitarios

\begin{tabular}{|c|c|c|}
\hline Maltrato & $\begin{array}{c}\text { Mujeres } \\
\mathbf{\%}\end{array}$ & $\begin{array}{c}\text { Hombres } \\
\mathbf{\%}\end{array}$ \\
\hline Recibió violencia física regularmente & 23.0 & 27.9 \\
\hline $\begin{array}{c}\text { Recibió violencia } \\
\text { psicológicaregularmente }\end{array}$ & 25.8 & 28.1 \\
\hline
\end{tabular}

Fuente: Elaboración Propia

En la tabla 3 se observa que los hombres reportarían recibir más violencia tanto física como psicológica de parte de sus padres respecto de sus pares mujeres. La violencia psicológica es las más reportadas tanto en los hombres como en las mujeres $28,1 \%$ y $25,8 \%$ respectivamente. Pero muy cercana a la física donde reportan $27,9 \%$ en los hombre y $23 \%$ en las mujeres.

Tabla 4. Violencia de género/maltrato recibido y su relación en la prevalencia de violencia psicológica recibida en relaciones de parejas.

\begin{tabular}{|c|c|c|c|c|}
\hline \multirow{2}{*}{ Violencia de género } & \multicolumn{2}{|c|}{$\begin{array}{l}\text { Violencia en relación de } \\
\text { pareja actual }\end{array}$} & \multirow[b]{2}{*}{$\mathrm{t}$} & \multirow[b]{2}{*}{$\mathrm{p}$} \\
\hline & $\begin{array}{l}\text { SÍ - Media } \\
\text { violencia } \\
\text { psicológica }\end{array}$ & $\begin{array}{l}\text { NO -Media } \\
\text { violencia } \\
\text { psicológica }\end{array}$ & & \\
\hline Existió violencia física & (M) 20,06 & 14,05 & 2,086 &, $03 * * *$ \\
\hline Vio o escucho violencia física. & (M) 17,51 & 14,64 & 1,002 & 31 \\
\hline Existió violencia psicológica & (M) 16,67 & 13,31 & 1,322 &, 18 \\
\hline Vio o escucho violencia psicológica & (M) 16,49 & 13,87 &, 985 & 32 \\
\hline Existió violencia física & (H) 14,45 & 11,30 & ,988 & 32 \\
\hline Vio o escucho violencia física. & $(\mathrm{H}) 13,65$ & 11,67 &, 656 & 51 \\
\hline Existióviolencia psicológica. & (H ) 14,14 & 10,47 & 1,424 &, 15 \\
\hline Vio o escuchoviolencia psicológica & (H) 16,03 & 9,49 & 2,570 &, $01 * *$ \\
\hline \multicolumn{5}{|l|}{ Maltrato recibido de los padres } \\
\hline Recibió maltrato físico regularmente & (M) 20,73 & 13,91 & 2,408 &, $01 * *$ \\
\hline Recibió maltrato psicológico regularmente & (M) 21,02 & 13,31 & 2,852 &, $00 * *$ \\
\hline Recibió maltrato físico regularmente & (H) 13,91 & 11,34 & ,993 &, 32 \\
\hline Recibió violencia psicológica regularmente & $(\mathrm{H}) 16,15$ & 10,40 & 2,242 &, $02 * * *$ \\
\hline
\end{tabular}

Fuente: Elaboración propia: $* * \mathrm{P}<0,01 * * * \mathrm{P}<0,05$ ( $\mathrm{M}=$ mujer; $\mathrm{H}=$ hombre)

En la Tabla 4 se observa que en el caso de las mujeres, presentan promedios más altos y estadísticamente significativos en el puntaje de violencia psicológica recibida en sus relaciones de pareja cuando existió violencia física hacia la madre, $(M=20,06 ; M=14,05, P=0,03)$ cuando recibió maltrato físico regularmente $(\mathrm{M}=20,73$; $\mathrm{M}=13,91, \mathrm{p}=0,01)$ y cuando recibió maltrato psicológico regularmente $(\mathrm{M}=21,02 ; \mathrm{M}=13,3, \mathrm{p}=0,00)$ respecto de las mujeres que no tuvieron la experiencia de violencia de género o maltrato. En el caso de los hombres, estos presentan promedios más altos y estadísticamente significativos en el puntaje de violencia psicológica recibida en sus relaciones de pareja cuando vieron o escucharon violencia de género de tipo psicológica hacia la madre $(\mathrm{H}=16,03 ; \mathrm{M}=9,49, \mathrm{p}=0,01)$ y cuando recibieron maltrato psicológico regularmente por parte de padres $(H=16,15, H=10,4, p=0,02)$, respecto de los jóvenes que no tuvieron la experiencia de violencia de género o maltrato 
Tabla 5. Presencia de violencia de género/ maltrato recibido y su relación en la prevalencia de violencia de tipo emocional recibida en las relaciones de parejas

\begin{tabular}{|c|c|c|c|c|}
\hline \multirow[b]{2}{*}{ Violencia de género } & \multicolumn{2}{|c|}{$\begin{array}{c}\text { Violencia en relación de } \\
\text { pareja actual }\end{array}$} & \multirow[b]{2}{*}{$\mathrm{t}$} & \multirow[b]{2}{*}{$\mathrm{p}$} \\
\hline & $\begin{array}{l}\text { SÍ - Media } \\
\text { violencia } \\
\text { emocional }\end{array}$ & $\begin{array}{l}\text { NO - Media } \\
\text { violencia } \\
\text { emocional }\end{array}$ & & \\
\hline Existió violencia física. & $(\mathrm{M}) 2,21$ & 1,54 & 1,036 & 30 \\
\hline Vio o escucho violencia física & (M) 2,04 & 1,56 &, 732 & 46 \\
\hline Existió violencia Psicológica & (M) 1,83 & 1,58 &, 412 &, 68 \\
\hline Vio o escucho violenciaPsicológica & (M) 2,24 & 1,43 & 1,314 &, 19 \\
\hline Existió Violencia Física & $(\mathrm{H}), 84$ &, 72 & 289 &, 77 \\
\hline Vio o escucho violencia física & $(\mathrm{H}), 77$ &, 75 &, 049 & ,96 \\
\hline Existió violencia Psicológica & (H) 1,14 &, 56 & 1,654 &, 10 \\
\hline Vio o escuchoviolencia Psicológica & $(\mathrm{H}) 1,32$ & 34 & 3,287 &, $00 * *$ \\
\hline \multicolumn{5}{|l|}{ Maltrato recibido desde los padres } \\
\hline Recibió maltrato físicoregularmente & (M) 2,00 & 1,56 &, 689 & 49 \\
\hline Recibió maltrato psicológico regularmente & $(\mathrm{M}) 2,58$ & 1,33 & 2,061 &, $04 * * *$ \\
\hline Recibió maltrato físico regularmente & $(\mathrm{H}), 72$ & 69 &, 075 & ,94 \\
\hline Recibió maltrato psicológico regularmente & $(\mathrm{H}), 88$ & 62 &, 764 & 244 \\
\hline
\end{tabular}

Fuente: Elaboración Propia:** $\mathrm{P}<0,01 * * * \mathrm{P}<0,05(\mathrm{H}=$ hombre; $\mathrm{M}=$ Mujer)

Se observa en la tabla 5, que cuando las mujeres señalan recibir maltrato psicológico regularmente durante la infancia, presentan un promedio más alto en violencia emocional en sus relaciones de pareja respecto a quienes no la declaran $(\mathrm{M}=2,58 ; \mathrm{M}=1,33, \mathrm{p}=0,049)$. En el caso de los hombres, cuando declaran haber visto o escuchado violencia psicológica hacia la madre, presentan un promedio más alto y estadísticamente significativo en la dimensión violencia emocional en sus relaciones de parejas que los varones que no la declaran ( $M=1,32$; $\mathrm{M}=0,34, \mathrm{p}=000)$.

Tabla 6. Exposición a violencia de género y maltrato recibido / su relación en la prevalencia de violencia física recibida en las relaciones de parejas.

\begin{tabular}{|c|c|c|c|c|}
\hline \multirow[b]{2}{*}{ Violencia de género } & \multicolumn{2}{|c|}{$\begin{array}{c}\text { Violencia en relación de pareja } \\
\text { actual }\end{array}$} & \multirow[b]{2}{*}{$\mathrm{t}$} & \multirow[b]{2}{*}{$\mathrm{p}$} \\
\hline & $\begin{array}{c}\text { SÍ - Media } \\
\text { violencia física }\end{array}$ & $\begin{array}{l}\text { NO - Media } \\
\text { violencia fisica }\end{array}$ & & \\
\hline Existió violencia física & (M), 98 & 67 & 1,127 & 26 \\
\hline Vio o escucho violencia física & (M), 78 &, 70 & 327 &, 74 \\
\hline Existió violencia Psicológica & (M), 73 & 66 & ,305 &, 76 \\
\hline VioPsicológica & (M),84 & 64 & 830 & 40 \\
\hline Existió violencia física & $(\mathrm{H}), 42$ &, 31 & 455 & 65 \\
\hline Vio o escucho violencia física & (H), 45 & 34 & 475 & 63 \\
\hline Existió violencia Psicológica & $(\mathrm{H}), 61$ & .21 & 1,906 &, $05^{* * *}$ \\
\hline Vio o escuchoviolencia Psicológica & $(\mathrm{H}), 68$ &, 19 & 2,300 &, $02 * * *$ \\
\hline \multicolumn{5}{|l|}{ Maltrato recibido por los padres } \\
\hline Recibió maltrato fisico regularmente & $(\mathrm{M}) 1,06$ & 66 & 1,501 &, 13 \\
\hline $\begin{array}{l}\text { Recibió maltrato psicológico } \\
\text { regularmente }\end{array}$ & (M) 1,05 &, 63 & 1,638 &, 10 \\
\hline Recibió maltrato físico regularmente & (H), 38 &, 35 & 108 & 91 \\
\hline $\begin{array}{l}\text { Recibió maltrato psicológico } \\
\text { regularmente }\end{array}$ & (M), 58 & 26 & 1,517 &, 13 \\
\hline
\end{tabular}

Fuente: Elaboración propia: ${ }^{* *} \mathrm{P}<0,01 * * * \mathrm{P}<0,05(\mathrm{M}=$ mujer; $\mathrm{H}=$ hombre) 
En la tabla 6, se observa que en el caso de las mujeres no existen diferencias significativas en los promedios de violencia física recibida en sus relaciones de pareja, entre quienes declaran violencia de género o maltrato infantil y los que no lo hacen. En los hombres se observan diferencias estadísticamente significativas entre quienes declaran la existencia de violencia psicológica del padre a la madre y quienes no en relación a la presencia de violencia física recibida durante sus relaciones de pareja $(\mathrm{M}=0,61 ; \mathrm{M}=0,21, \mathrm{p}=0,05)$.Situación similar ocurre cuando se declara haber visto o escuchado violencia de genero de tipo psicológica, donde los promedios de los hombres que la señalan, son más altos respecto a recibir violencia física durante sus relaciones de $\operatorname{parejas}(\mathrm{M}=0,60 ; \mathrm{M}=, 019, \mathrm{p}=0,02)$.

Tabla 7. Exposición a violencia de género y maltrato infantil recibido / su relación en la prevalencia de violencia psicológica ejercida en las relaciones de pareja.

\begin{tabular}{|c|c|c|c|c|}
\hline \multirow[b]{2}{*}{ Violencia de género } & \multicolumn{2}{|c|}{$\begin{array}{c}\text { Violencia en relación de } \\
\text { pareja actual }\end{array}$} & \multirow[b]{2}{*}{$\mathrm{t}$} & \multirow[b]{2}{*}{$\mathrm{p}$} \\
\hline & $\begin{array}{l}\text { Sí - Media } \\
\text { violencia } \\
\text { psicológica }\end{array}$ & $\begin{array}{c}\text { NO - Media } \\
\text { violencia } \\
\text { psicológica }\end{array}$ & & \\
\hline Existió violencia física. & (M)15,46 & 12,12 & 1,500 &, 13 \\
\hline Vio o escucho violencia física & (M)14,45 & 12,42 & 911 & 36 \\
\hline Existió violencia Psicológica & (M)13,97 & 11,57 & 1,181 & 23 \\
\hline Vioo escucho violencia psicológica & (M)14,57 & 11,29 & 1,579 &, 11 \\
\hline Existió Violencia física & (H) 10,45 & 7,98 & 1,223 & 22 \\
\hline Vio o escucho violencia física. & (H) 10,48 & 7,94 & 1,345 &, 18 \\
\hline Existió violencia psicológica. & (H) 9,43 & 7,46 & 1,232 & 22 \\
\hline Vio o escucho violencia psicológica & (H) 10,86 & 6,76 & 2,590 &, $01^{* *}$ \\
\hline \multicolumn{5}{|l|}{ Maltrato recibido por los padres } \\
\hline Recibió maltrato físico regularmente & (M) 17,04 & 11,87 & 2,370 &, $01^{* *}$ \\
\hline Recibió maltrato psicológico regularmente & (M) 17,0 & 11,52 & 2,623 &, $00^{* *}$ \\
\hline Recibió maltrato físico regularmente & (H) 9,97 & 7,63 & 1,414 &, 16 \\
\hline Recibió maltrato psicológico regularmente & (H) 11,2 & 7,08 & 2,470 &, $01^{* \star *}$ \\
\hline
\end{tabular}

Elaboración propia:**P<0,01 ***P<0,05 (M=mujer; $\mathrm{H}=$ Hombre)

En la tabla 7 se observa que las mujeres que señalaron recibir maltrato físico $(\mathrm{M}=17,04 ; \mathrm{M}=11,52$, $\mathrm{p}=0,01)$ y psicológico regularmente $(\mathrm{M}=17,0 ; \mathrm{M}=11,52, \mathrm{p}=0,00)$ desde sus padres, presentan medias más altas y estadísticamente significativas en ejercer violencia psicológica a sus parejas de que quienes no declararon su presencia. En el caso de los hombres, quienes señalaron ver o escuchar psicológica a sus madres $(H=11,2 ; H=7,08, p=0,01)$ y recibir maltrato psicológico regularmente durante la infancia, $(H=11,2$; $\mathrm{H}=7,08, \mathrm{p}=0,01)$ presentan medias más altas y estadísticamente significativas de violencia física recibida que quienes no lo declararon. 
Tabla 8. Exposición a la violencia de género y maltrato infantil y su relación en la prevalencia de violencia emocional ejercida en sus relaciones de pareja.

\begin{tabular}{|c|c|c|c|c|}
\hline \multirow[b]{2}{*}{ Violencia de genero } & \multicolumn{2}{|c|}{$\begin{array}{c}\text { Violencia en relación de } \\
\text { pareja actual }\end{array}$} & \multirow[b]{2}{*}{$\mathrm{t}$} & \multirow[b]{2}{*}{$\mathrm{p}$} \\
\hline & $\begin{array}{l}\text { Sí - Media } \\
\text { violencia } \\
\text { emocional }\end{array}$ & $\begin{array}{c}\text { NO - Media } \\
\text { violencia } \\
\text { emocional }\end{array}$ & & \\
\hline Existió Violencia física. & (M) 1,21 & 82 & 848 & 39 \\
\hline Vio o escucho violencia física. & $(\mathrm{M}), 86$ & 93 &,- 138 & 89 \\
\hline Existió violencia psicológica. & $(\mathrm{M}), 76$ & 1,01 &,- 583 &, 56 \\
\hline vio o escucho violenciapsicológica & (M) 1,16 &, 79 &, 826 & 41 \\
\hline Existió Violencia física & (H), 67 &, 36 & 987 & 32 \\
\hline Vio o escucho violencia física & (H),, 86 &, 35 & 1,763 &, 08 \\
\hline Existió violencia psicológica & (H), 77 &, 30 & 1,912 & $05^{\star \star * *}$ \\
\hline Vioo escucho violencia psicológica & (H),, 85 &, 16 & 3,038 &, $00^{* *}$ \\
\hline \multicolumn{5}{|l|}{ Maltrato recibido } \\
\hline Recibió maltrato físico regularmente & (M) 1,04 & 85 & 418 & 67 \\
\hline $\begin{array}{l}\text { Recibió maltrato psicológico } \\
\text { regularmente. }\end{array}$ & (M) 1,40 & .69 & 1,641 &, 10 \\
\hline Recibió maltrato físico regularmente & (H), 66 &, 32 & 1,400 &, 16 \\
\hline $\begin{array}{l}\text { Recibiómaltrato psicológico } \\
\text { regularmente. }\end{array}$ & (H), 45 &, 37 &, 348 &, 72 \\
\hline
\end{tabular}

Elaboración propia $* * \mathrm{P}<0,01 * * * \mathrm{P}<0,05(\mathrm{M}=$ mujer, $\mathrm{H}=$ Hombre)

En la tabla 8, se observa en el caso de las mujeres que los promedios en la subescala de violencia emocional ejercida en la pareja actual de las personas que declararon violencia de género y quienes no la declararon no presentan diferencias estadísticamente significativas. En el caso de los hombres, se aprecia que quienes señalaron la existencia $(\mathrm{H}=0,77 ; \mathrm{H}=0,30 ; \mathrm{p}=0,05)$ o que vieron o escucharon de violencia psicológica hacía la madre $(\mathrm{H}=0,85 ; \mathrm{H}=0,16 ; \mathrm{p}=0,00)$ presentaron medias más altas y estadísticamente significativas en violencia emocional ejercida respecto de los hombres que no la declararon.

Tabla 9. Exposición a violencia de género y maltrato recibido / su relación en la prevalencia de violencia física ejercida en relaciones de pareja.

\begin{tabular}{|c|c|c|c|c|}
\hline \multirow[b]{2}{*}{$\begin{array}{l}\text { Exposición a violencia de genero } \\
\text { en la niñez }\end{array}$} & \multicolumn{2}{|c|}{$\begin{array}{c}\text { Violencia en relación de } \\
\text { pareja actual }\end{array}$} & \multirow[b]{2}{*}{$\mathrm{t}$} & \multirow[b]{2}{*}{$\mathrm{p}$} \\
\hline & $\begin{array}{c}\text { SÍ - Media } \\
\text { violencia } \\
\text { física }\end{array}$ & $\begin{array}{c}\text { NO - Media } \\
\text { violencia } \\
\text { física }\end{array}$ & & \\
\hline Existió violencia física & (M) 1,13 &, 68 & 1,687 &, 09 \\
\hline Vio o escucho violencia física & $(\mathrm{M}), 94$ &, 74 &, 729 & 46 \\
\hline Existió violencia Psicológica & $(\mathrm{M}), 88$ &, 68 & 832 & 40 \\
\hline Vio o escucho violenciaPsicológica. & (M) 1,06 & .59 & 1,849 & .06 \\
\hline Existió Violencia física & (H), 32 & 25 & 311 &, 75 \\
\hline Vio o escucho violencia física. & (H), 45 & 24 & 1,098 & 27 \\
\hline Existió violencia Psicológica. & (H),, 56 &, 14 & 2,462 &, $01 * *$ \\
\hline Vio o escucho violenciaPsicológica. & (H) ,41 &, 12 & 2,011 &, $04^{* * *}$ \\
\hline \multicolumn{5}{|l|}{ Maltrato recibido en la niñez } \\
\hline Recibió maltrato físico regularmente & $(\mathrm{M}), 98$ &, 74 &, 895 & 37 \\
\hline Recibió maltrato psicológico regularmente & (M)1,11 & 67 & 1,708 &, 08 \\
\hline Recibió maltratofísico regularmente & (H), 47 & 20 & 1,666 & .09 \\
\hline Recibió maltrato psicológica regularmente & (H), 48 &, 15 & 2,182 &, $03^{* \pi *}$ \\
\hline
\end{tabular}

Elaboración propia $* * \mathrm{P}<0,01 * * * \mathrm{P}<0,05$ (M= mujer, $\mathrm{H}=$ Hombre) 
En la tabla 9, se observa en el caso de las mujeres, que los promedios de violencia física ejercida en la pareja actual de las que declararon violencia de género y quienes no la declararon no presentan diferencias estadísticamente significativas. En los hombres, en cambio, se aprecia que los que declararon la existencia de violencia de género de tipo psicológica $(\mathrm{H}=0,56 ; \mathrm{H}=0,14 ; \mathrm{p}=0,01)$ y que escucharon o vieron este tipo de violencia $(\mathrm{H}=0,41 ; \mathrm{H}=0,12 ; \mathrm{p}=0,04)$ presentaron medias más altas y estadísticamente significativas en violencia física ejercida respecto de los hombres que no la declararon. Finalmente, los hombres que señalaron que recibir maltrato psicológico regularmente desde sus padres $(H=0,48 ; H=0,15 ; p=0,03)$ presentó medias más alta y estadísticamente significativa de los hombres que no lo manifestaron.

\section{Discusión}

En cuanto a la violencia de género, las mujeres percibieron mayor frecuencia de historia de violencia de este tipo, en relación a sus pares hombres, no obstante, los hombres presentaron mayor reporte de maltrato tanto físico como psicológico desde los padres hacía ellos.

La violencia de género más reportada fue la violencia psicológica, ya sea reportándose su existencia como de haberla visto o escuchado, tanto de los jóvenes hombres como de las mujeres. Éstas últimas presentaron mayor reporte de esta violencia. Lo mismo ocurre con la existencia de violencia de género de tipo física que también fue levemente más reportada en las adolescentes mujeres que en los varones, con una diferencia de 8 puntos porcentuales, y de si vio o escucho violencia física con una diferencia de 5 puntos porcentuales más que los hombres.

En cuanto al maltrato los varones reportaron recibir en la niñez violencia psicológica y física regularmente más que sus pares mujeres. Según la United Nations (2006); Save the Children (2007) la mayor frecuencia del castigo físico es hacia los niños y el abuso psicológico a las niñas al obligarlas a cumplir ciertos patrones de comportamiento culturalmente aceptados. Estos datos son similares a los estudios de Ross (1996) y Wauchope y Strauss, (1990). Los infantes y adolescentes hombres tienen más riesgos de ser castigados físicamente que los de sexo femenino. En todos los contextos y tipos de exposición los hombres perciben más violencia que las mujeres (Vásquez, et al, 2018). En nuestro estudio tanto hombres como mujeres reportaron exposición a violencia de género y maltrato, pero en este último levemente más los hombres que las mujeres, no obstante ambos son objeto de maltrato de sus padres en porcentajes sobre el $20 \%$.

En cuanto a ser víctimas de violencia en las relaciones de pareja, la experiencia de existencia over y/o escuchar violencia psicológica del padre a la madre resultó ser significativa para los adolescentes hombres para recibir violencia de tipo física, psicológica y emocional en sus relaciones de pareja. Para las adolescentes mujeres observar violencia de género de tipo psicológica no se relacionó significativamente para recibir ningún tipo de violencia en el noviazgo. En cuanto a la experiencia de violencia de género de tipo física resultó significativa para recibir violencia psicológica en las adolescentes mujeres en sus relaciones.

En cuanto a ejercer violencia en sus relaciones de algún tipo, la experiencia de existencia de violencia psicológica o ver o escucharla se relacionó significativamente con ejercer violencia de tipo psicológica, emocional y física hacia sus parejas en sus relaciones de parejas desde los hombres. En el caso de las mujeres la exposición de violencia de género de cualquier tipo no se relacionó con ejercer ningún tipo de violencia en sus relaciones.

Una hipótesis que las investigaciones en este tema plantea que los niños aprenderían que la violencia es una estrategia eficaz de resolución de problemas y que en las niñas esta violencia las llevaría a adoptar conductas de sumisión y obediencia (Sarasúa, et al, 1996). Para otros, como Kwong, et al. (2003), todas las formas de violencia en la familia fueron predictivas de todas las formas de abuso en la relaciones de pareja, además no encontraron patrones específicos respecto del género y tampoco lograron relacionar la perpetración con los hombres y la victimización con las mujeres. Nuestros hallazgos respaldan la afirmación de que la exposición a la violencia de género se asocia con la perpetración física, emocional y psicológica de la violencia en el noviazgo desde los hombres, siendo la de mayor peso la violencia psicológica que observan o escuchan en sus familias 
de origen. Los estudios respaldan la hipótesis de la violencia física del padre a la madre influiría en la perpetración de abuso psicológico en el noviazgo (Kwong et. al, 2003). En nuestro estudio, para los hombres la exposición a cualquier experiencia de violencia física no se relaciona con ninguna violencia en las relaciones de parejas, y para el caso de las mujeres ver violencia física se relaciona con recibir violencia psicológica en el pololeo.

En cuanto al maltrato de los padres y ser víctima de violencia en las relaciones de parejas, en el caso de los adolescentes hombres el recibir maltrato psicológico regularmente de los padres resultó significativo para recibir violencia psicológica y ejercer violencia de tipo física y psicológica en sus relaciones. En el caso de las adolescentes mujeres, se relacionó el recibir maltrato psicológico regularmente de los padres con recibir violencia psicológica y emocional en sus relaciones de pareja, junto con ejercer violencia psicológica en las mismas.

Para el caso de maltrato físico desde los padres a las adolescentes mujeres, resultó significativa para recibir violencia psicológica y ejercer violencia psicológica en sus relaciones de pareja. El maltrato físico hacia los hombres no se relacionó significativamente con el ejercicio de perpetración o victimización en sus relaciones.

En este estudio tanto para hombres como para mujeres el recibir maltrato de los padres se relacionó con recibir y ejercer violencia en las relaciones de parejas. Marshall y Rose (1988) encontraron que la violencia familiar de padres a hijos predice la perpetración y la victimización de los hombres, pero solo la victimización de las mujeres.

Figura 1. Resumen de la relación entre violencia de genero/maltrato y su relación con la violencia de genero recibida o ejercida.

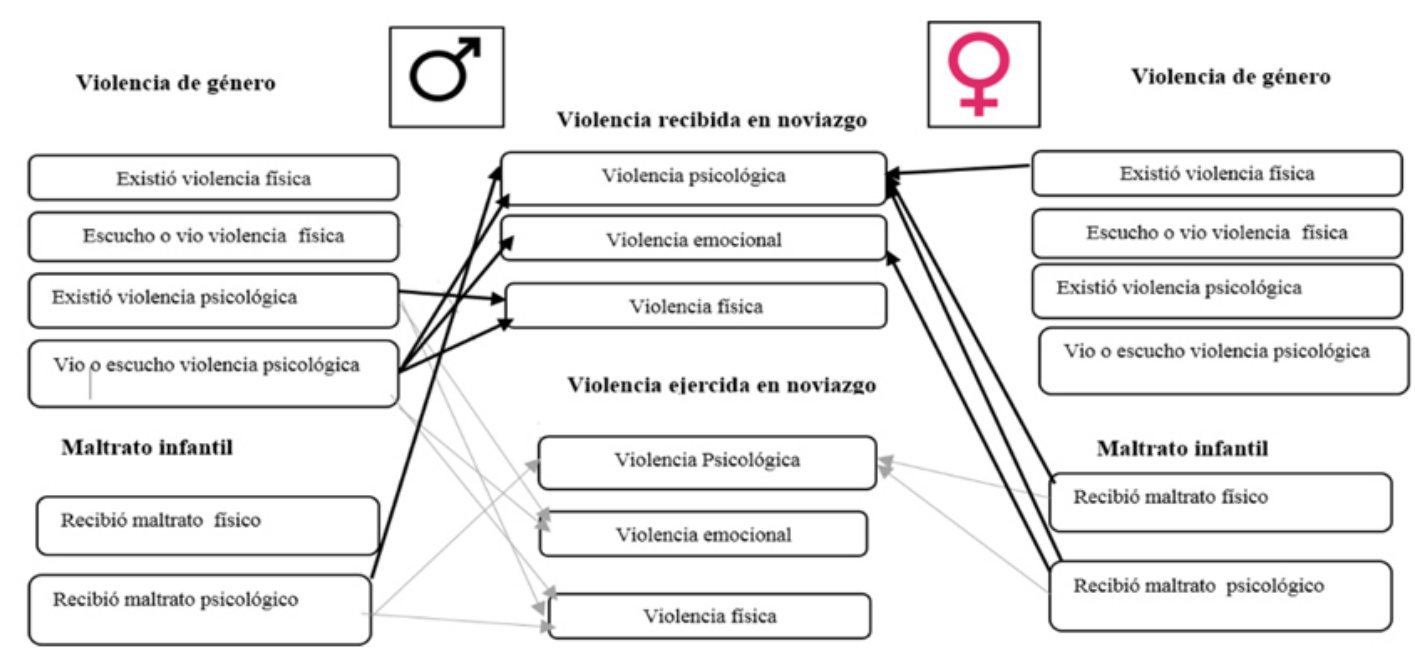

Elaboración Propia(violencia recibida)(-violencia ejercida)

Como se puede ver en la Figura 1,la violencia de género de tipo psicológica influye significativamente en los adolescentes hombres tanto para ser víctima de violencia como para ejercer violencia en sus relaciones en los tres tipos de violencia de pareja estudiados. En cambio en las mujeres lo que más les influiría es el maltrato infantil, esto les haría más víctimas de violencia psicológica y emocional en su relación de pareja y ejercer violencia psicológica a sus parejas. La violencia de género vista hacia sus madres solo le afectaría la de tipo física pero en ser víctima de violencia psicológica en el noviazgo, pero no para ser perpetradoras de violencia en sus parejas.

Al tratar de explicarnos los resultados, algunos estudios(Alvarado et al., 1998; Heise, 1994;Morrison y Orlando, 1999) señalan que las hijas de mujeres víctimas de violencia doméstica tienen mayores probabilidades 
de sufrir ese tipo de agresiones, y los hijos de esas mujeres son más propensos a perpetrarla. En nuestro estudio la relación entre violencia de pareja (ejercida y recibida) y la experiencia de violencia en la familia de origen se encuentra más patente en el caso de los hombres que de las mujeres.

Una de las críticas en los estudios cuantitativos de los autoinformes es la alta deseabilidad social de las respuestas tanto en hombres como mujeres (De las Cuevas y González de Rivera, 1992), sin olvidar la culpabilización, negación o minimización de las conductas violentas (Scott y Straus, 2007).

Los hombres tuvieron una mayor asociación la historia de violencia de género con ejercer violencia que las mujeres. Whitfield et al, (2003), evaluaron a 8.629 niños/as expuestos a violencia de género, en particular el maltrato físico hacia la madre; los resultados señalan que los menores expuestos eran más propensos a emplear la violencia en la pareja intima en la edad adulta que los no expuestos, mientras que las niñas tenían más probabilidades de ser víctimas. Kwong, et, al,(2003)concluyeron que el abuso psicológico en la niñez explica mejor el abuso físico en las relaciones violentas, lo cual coincidiría con los resultados del presente estudio, pero no logra establecer una diferencia de género, que en este estudio esa relación solo apunta a los hombres. Además, hay evidencia de que el abuso emocional, en comparación con el abuso físico, en la experiencia de violencia en los padres, puede tener un efecto devastador, si no más, en los jóvenes, (Follingstad, et al, 1990)lo que es coincidente con nuestros resultados.

No hay una relación directa entre recibir o estar expuesto a un tipo de violencia de género y ejercer el mismo en la relaciones de noviazgo. Pero si podemos señalar que las formas de abuso psicológico y físico se asocian con un mayor riesgo de involucrarse en relaciones psicológicas abusivas tanto en hombres como en mujeres. La violencia de género o maltrato repercute tanto en la victimización como perpetración. En lo específico cabe hacerse la pregunta de ¿por qué la violencia psicológica pareciera ser más relevante que la física para el caso de los hombres? y ¿por qué la violencia de género observada en sus familias no se asocia con recibir o ejercer violencia en la pareja en el caso de las mujeres?

El estudio tiene implicaciones importantes para la prevención primaria y secundaria en el noviazgo, pues explica al menos parcialmente que la violencia de género y maltrato aumentan el riesgo de implicarse en la violencia en el pololeo o noviazgo. Los programas de prevención secundaria dirigido a niños o adolescentes que han vivido violencia de género y maltrato no solo prevendría la violencia en las primeras relaciones de noviazgo o pololeo, sino también la violencia de género en la vida adulta. Además la eficacia de esos programas deben considerar las necesidades únicas de cada género pues la re significación parece ser muy distinta. Es necesario analizar también el propio proceso de construcción de la experiencia pasada, pues generalmente será en sus estructuras simbólicas, culturales, donde se puedan encontrar las claves que permitan entender por qué algunas personas repiten las pautas de socialización y normalización de la violencia observada y normalizada y otras no, y cuáles son la factores protectores o mediadores que permitan incorporarlos en la intervención profesional. Los cambios, por ende, deberían considerar la cultura que permite la naturalización de la violencia, por lo que se hace urgente y necesario trabajar en la reconstrucción de los estereotipos de género desde el interior de la familia y en todas las instituciones que permiten su reproducción binaria y jerarquizada. El sistema social, como tal, ha ido generando formas o estrategias para seguir el orden y mantener la distribución del poder, y la violencia es una de las formas más frecuentes utilizadas para lograrlo, y que sigue instituyéndose como una forma de relación incorporada al repertorio social. La violencia hacia la mujer repercute en el bienestar de todos los otros miembros de la familia, en la vida comunitaria y que va multiplicando las consecuencias del fenómeno.

La presente investigación con estudiantes de educación superior nos señala que el nivel educacional formal no es un factor protector de la violencia. Este estudio no pretende ser determinista, al contrario, sus resultados nos ayudan a mostrar la necesidad de prevención en instituciones como la familia y comunidad. Al mismo tiempo se visualiza la necesidad y responsabilidad que tienen las instituciones de educación superior en el abordaje de esta temática, ya sea incorporándola en las mallas curriculares o generando programas preventivos que aborden sistemáticamente las experiencias de violencia y promuevan nuevas formas de concebir las relaciones de pareja. 


\section{Agradecimientos}

Los siguientes resultados de investigación son parte del proceso investigativo llevado a cabo por la autora Soraya Espinoza Moragaque se encuentra adscrita al programa de doctorado en Trabajo Social de la Universidad Complutense de Madrid España y con el apoyode la Universidad De los Lagos Chile

\section{Bibliografía}

Alvarado, G., Moysen, J., Estrada, S. y Terrones, A. (1998). Prevalencia de violencia doméstica en la ciudad de Durango. Salud Publica de México 40 (6), 481-486

Andrés, A., López, S. y Álvarez, E. (2008). Valoración del riesgo de violencia contra la pareja por medio del SARA. Papeles del psicólogo, 29, 107-122.

Barnett, O., Miller-Perrin, C., yPerrin, R. (1997). Family violence across the lifespan: An introduction. Thousand Oaks, CA, UK: Sage.

Bernard, M. y Bernard, J. (1983). Violent intimacy: The family as a model for love relationships. Family Relations. 32, 283-286.

Bourdieu, P. (1998).La dominación masculina. Paris, France: Editions du Seuil

Brañes, P. (2006). Violencia de genero. La Paz, Bolivia: CIDEM (Centro de Información y Desarrollo de la Mujer Bolivia).

Cascardi, M., y Jouriles, E. (2018). Mechanims underlying the association of exposure to family of origin violence and adolescent dating violenceEn D.Wolfe y J. Temple (Eds),Adolescent dating violence- Theory Research, an prevention. (pp.159-188). London, United Kingdom: Academic Press

Centers for Disease Control and Prevention. (2006). Physical dating violence among High School students, United States, 2003. Morbidity and Mortality Weekly Report, 55 (19), 532-535

Close, S. (2005). Dating violence prevention in middle school and high school youth. Journal of Child and Adolescent Psychiatric Nursing, 18(1), 2-9

Consejo de Europa (2010). Children who Witness Domestic Violence, informe de Carina Ohlsonmiembro del grupo socialista de la Asamblea Parlamentaria del Consejo de Europa, 2010. Recuperado de: http:// assembly.coe.int/Main.asp?link=/Documents/WorkingDocs/Doc10/

EDOC12155.htm

Corral, S. (2009). Estudios de la violencia en el noviazgo en jóvenes universitarios/as: cronicidad, severidad y mutualidad de las conductas violentas. Psicopatología Clínica Legal y Forense, 9, 29-48

De las Cuevas, C. y González de Rivera, J. (1992). Autoinformes y respuestas sesgadas. Anales de Psiquiatría, 8(9), 362-366.

DuMont, K., Widom, C. yCzaja, S. (2007). Predictors of resilience in abused and neglected children grown-up: The role of individual and neighborhood characteristics. Child Abuse \& Neglect, 31, 255-274

Edleson, J. (1999). Children's witnessing of adult domestic violence. Journal of Interpersonal Violence, 14, 839-870.

Fernández-Montalvo, J. y Echeburúa, E. (1997). Variables psicopatológicas y distorsiones cognitivas de los maltratadores en el hogar: un análisis descriptivo. Análisis y Modificación de Conducta, 23, 151-180 
Follingstad, D., Rutledge, L., Berg, B., Hause, E. y Polek, D. (1990). The role of emotional abuse in physically abusive relationships. Journal of Family Violence, 5 (2):107-120.

Foshee, V., Bauman, K. y Linder, G. (1999). Family violence and the perpetration of adolescent dating violence: Examining social learning and social control proccesess. Journal of Marriage and the Family, 61 (2), 331342

Gálligo, E.(2009). SOS...Mi chico me pega pero yo le quiero. Madrid, España: Pirámide.

González, R. y Santana, J. (2001). La violencia en parejas jóvenes. Psicothema,12 (1),127-131

Graham-Bermann, S., Grubera, G., Howell, K., y Girz, L. (2009). Factors discriminating among profiles of resilience and psychopathology in children exposed to intimate partner violence (IPV). Child Abuse \& Neglect, 33, 648-660.

Guite, J. (2001). Adolescent battering relationships: A qualitive study of the female's experience. Dissertation Abstracts International Section-B: The Sciences and Engineering. 62(3-B), 1577.

Heise L. (1994). Violencia contra la mujer: la carga oculta sobre la salud. Programa Mujer, Salud y Desarrollo. Washington, DC, Estados Unidos: OPS

Hotaling, G., y Sugarman, D.(1986) An analysis of risk markers in husband to wife violence: The current state of Knowledge.Violence and victims 1 (2) 101-124

International Save the Children Alliance (2007). Children and Gender-based Violence: an overview of existing conceptual frameworks. London, UK: Save the Children

Kaukinen, C. (2014). Dating Violence Among College Students. Trauma, Violence, \& Abuse 15:4, 283-296.

Kitzmann, K., Gaylord, N., Holt, A. y Kenny, E. (2003). Child witnesses to domestic violence: a meta-analytic review. Journal of Consulting and Clinical Psychology, 71, 339?352.

Klevens, J. (2001). Prevalencia y marcadores de riesgo entre mujeres que consultan la red pública de servicios de salud en Bogotá. Bogotá, Colombia: Secretaría Distrital de Salud.

Kwong, M., Bartholomew, K., Henderson, A.J.Z., y Trinke, S. (2003). The intergenerational transmission of relationship violence.Journal of Family Psychology, 17, 288-301

Langhinrichsen-Rohling, J., Neidig, P. y Thorn, G. (1995). Violent marriages: Gender differences in levels of current violence and past abuse. Journal of Family Violence, 10 (2), 159-176

Larraín, S. y Bascuñán, C. (2008). Maltrato infantil y relaciones familiares en Chile: Análisis comparativo. 19942000-2006. Revista chilena de pediatría, 79 (Supl. 1).

Lewis, S., y Fremouw, W. (2001). Dating violence: A critical review of the literature. Clinical Psychology Review, 21, 105-127

Lichter, E., y McCloskey, L. (2004). The effects of childhood exposure to marital violence on adolescent genderrole beliefs and dating violence. Psychology and WomenQuarterly, 28, 344-357.https://doi.org/10.1111/ j.1471-6402.2004.00151.x

Loise, T. (2009). Children's Exposure to Intimate Partner Violence (IPV): Challenging Assumptions about child protection practices. A thesis submitted in conformity with the requirements for the degree of Ph. D. FactorInwentash Faculty of Social Work. University of Toronto. 
Luthar, S., Cicchetti, D. yBecker, B. (2000). The construct of resilience: A critical evaluation and guidelines for future work. Child Development, 71 (3), 543-558.

McCloskey, L., y Walker, M. (2000). Posttraumatic stress in children exposed to family violence and single-event trauma. Journal of the American Academy of Child \& Adolescent Psychiatry, 39(1), 108-115.

McCloskey, L., (2011). The impacto of intimate partner violence on adolescents. En: S. Graham- Bermann \& A. Levendosky (Eds.). How Intimate Partner violence affects Children. Developmental Research, Case Studies, and Evidence-Based Intervention (pp.225-246). Washington DC, United States: American Psychological Association.

MacEwen, K. (1994). Refining the intergenerational transmission hypothesis. Journal of Interpersonal Violence, 9, 350-365.

McGloin, J. y Widom, C. (2001). Resilience among abused and neglected children grown up. Development and Psychopathology, 13, 1021?1038.

Magen, R.H. (1999). In the best interest of battered women: Reconceptualizing allegations of failure to protect. Child Maltreatment, 4, 127-135.

Marshall, L., y Rose, P. (1988). Family oforigin and courtship violence. Journal ofCounseling and Development, 55,414-418

Martínez, J., Vargas, R., yNovoa, M. (2016). Relación entre la violencia en el noviazgo y observación de modelos parentales de maltrato. Psychologia: Avances de la Disciplina, 10(1), 101-112.

Masten, A., Best, K. y Garmezy, N. (1991). Resilience and development: Contributions from the study of children who overcome adversity. Development and Psychopathology, 2, 425-444.

Matud, M., Marrero, R., Carballeira, M., Pérez, M., Correa, M., Aguilera, B. y Sánchez, T. (2003). Transmisión intergeneracional de la violencia doméstica. Psicología Conductual, 11 (1), 25-40.

Matud, M. (2007). Dating violence and domestic violence. Journal of Adolescent Health, 40, 295-297. (2012). Psicología del Género. Madrid, España: La Laguna

Makepeace, J. (1981). Courtship violence among college students. Family Relations, 30, 97-102.

Morrison, A. y Orlando, M. (1999). Socialandeconomic costs ofdomestic violence: Chile and Nicaragua. Washington DC, United States: Inter-American Development Bank, Johns Hopkins Press.

Meltzer, H., Doos, L., Vostanis, P., Ford, T. y Goodman, R. (2009). The mental health of children who witness domestic violence. Child \& Family Social Work, 14(4), 491-501. doi: 10.1111/j.1365-2206.2009.00633.x

Nunnally, Jum C. (1987).Teoría psicométrica. Ciudad de México, México: Trillas.

O’Leary, K., Barling, J., Arias, I., Rosenbaum, A., Malone, J. y Tyree, A. (1989). Prevalence and stability of physical aggression between spouses: a longitudinal analysis. J. Consult Clin Psychol, 547, 263-268.

ONU (Organización Naciones Unidas)(1993) Declaración sobre eliminación de la violencia contra la mujer. Resolución de la Asamblea 48/104 del 20 de diciembre de 1993

ONU (2006) Informe sobre violencia contra los niños y las niñas, del experto independiente Paulo Sérgio Pinheiro, Naciones Unidad 2006 (pp. 45-109). Recuperado de: http://www2.ohchr.org/english/bodies/crc/study.htm 
Osorio, S. G. (2013). ¿Por qué aún la violencia de género? Una respuesta conceptual a la persecución contra quienes no caben en las categorías “hombre” o “mujer”. Recuperado de: http:// manzanadiscordia.univalle.edu.co/ volúmenes/artículos/V8N1/art4.pdf

Paat, Y. F., y Markham, C. (2016). The Roles of Family Factors and Relationship Dynamics on Dating Violence Victimization and Perpetration Among College Men and Women in Emerging Adulthood. Journal of interpersonal violence, 34(1), 81-114. doi:10.1177/0886260516640544

Pederson, P. y Thomas, C. (1992). Prevalence and correlates of dating violence in a Canadian university sample. Canadian Journal of Behavioral Science, 24(4), 490-501.doi: 10.1037/h0078756

Rey-Anacona, C. (2015). Variables asociadas a los malos tratos en el noviazgo en adolescentes y adultos jóvenes. Acta Colombiana de Psicología, 18(1), 159-171.https://dx.doi.org/10.14718/ACP.2015.18.1.15

(2008)Prevalencia, factores de riesgo y problemáticas asociadas con la violencia en el noviazgo: una revisión literaria. RevistaAvances en Psicología Latinoamericana. 26(2), 227-241.

(2009). Maltrato en el noviazgo de tipo físico, psicológico, emocional, sexual y económico: un estudio exploratorio. Acta Colombiana de Psicología, 12(2), 27-36

Rivero, R., ySánchez, F., (2005) Determinants, effects and costs of domestic violence. Documento CEDE Ediciónelectrónica.

Rodríguez, Z. (1875). Diccionario de Chilenismos. Santiago: El Independiente. Recuperado de http:// www.memoriachilena.cl/ archivos2/pdfs/MC0012931.pdf

Ross, S. M. (1996). Risk of physical abuse to children of spouse abusing parents. Child Abuse \& Neglect, 20(7), 589-598. doi: 10.1016/0145-2134(96)00046-4

Rubiano, N., Hernández, A., Molina, C., Gutiérrez, M. y Vejarano, M. (2003). Conflicto y violencia intrafamiliar. Bogotá, Colombia: Universidad Externado de Colombia.

Rubio-Garay, F., López, M., Carrasco, M., y Amor, P., (2017). Prevalencia de la violencia en el noviazgo una revisión sistemática. Psychologist Papers, 38 (2), pp.135-147

Sarasúa, B., Zubizarreta, I., Echeburúa, E. y Corral, P. (1996). Perfil psicológico del maltratador a la mujer en el hogar. En E.Echeburúa (Dir.), Personalidades violentas (pp.111-128). Madrid, España: Pirámide.

SERNAM (2009) Análisis de la violencia en las relaciones de pareja entre jóvenes. Documento de trabajo N¹05.Santiago de Chile, 2009.Recuperado de:

<http://hivhealthclearinghouse.unesco.org/sites/default/files/resources/santiago_rec_20090318_112356.pdf >

Scott, K., y Straus, M. A. (2007). Denial, minimization, partner blaming and intimate aggression in dating partners. Journal of Interpersonal Violence, 22, 851-871.

Temched, C., Serbin, L., Martin-Storey, A., Stack, D., Hodgins, S., Ledingham, y Schwartzman, A. E. (2008). Continuity and pathways from aggression in childhood to family violence in adulthood: A 30-year longitudinal study. Journal of Family Violence, 23, 231-242. doi: 10.1007/s10896-007-9147-2

Unicef (2006). Behind closed doors: the impact of domestic violence on children. New York, United States: Unicef. Recuperado de: http://www.unicef.org/media/files/BehindClosedDoors.pdf.

Unicef(1989) Convención de los Derechos del Niño.Aprobada por Asamblea general de las Naciones Unidas 20 de noviembre de 1989. Ratificada por Chile en 1990. 
UNICEF y The body Shop International. Behind Closed Doors: The Impact of Domestic Violence on Children. Londres: UNICEF (Child Protection Section) y The Body Shop International Plc [Internet]; 2006. Recuperado de: www.unicef.org/protection/files/BehindClosedDoors.pdf.

United Nations General Assembly. (2006). Rights of the child. Note of the Secretary-General. Report of the independent expert for the United Nations study on violence against children.Geneva, Switzerland: United Nations. Recuperado de: https://www.unicef.org/violencestudy/ reports/SG_violencestudy_en.pdf

Vagi, K., Rothman, E., Latzman, N. Tharp, A. T., Hall, D. y Breiding, M. (2013). Beyond correlates: A review of risk and protective factors for adolescent dating violence perpetration. Journal of Youth and Adolescence, 42, 633-649. Doi:10.1007/s10964-013-9907-7

Vásquez, N., Gallego, F. y Bedregal, P. (2018). Diferencias en la percepción de violencia en niñas y niños de escuelas municipales y su asociación con actitudes parentales,Revista Ars Médicas3(1), 24-32. DOI: http:/ /dx.doi.org/10.11565/arsmed.v43i3.1152

Werner, E. y Smith, R. (1989). Vulnerable but invincible: A longitudinal study of resilient children and youth. New York, McGraw-Hill.

Whitfield, C., Anda, R., Dube, S. y Felitti, V. (2003). Adverse Childhood Experiences and Subsequent Hallucinations. Submitted, Child Abuse and Neglect.

Wolfe, D. A., Scott, K., Wekerle, C. y Pittman, A. (2001). Child maltreatment: Risk of adjustment problems and dating violence in adolescence. Journal of the American Academy of Child \& Adolescent Psychiatry. 40(3), 282-289.

Wauchope, B. yStraus, M (1990), Physical Punishment and Physical Abuse of American Children: Incidence Rates by Age, Gender and Occupational Class. En M. Straus y R. Gelles (eds.), Physical Violence in American Families. Risk Factors and Adaptations to Violence in 8145 Families. pp. 133-148, New Brunswick, Canada: Transaction publisher. 